sciforum

\title{
How to define the quality of images
}

\section{Adrian Carballal, Nereida Rodriguez, Iria Santos, Luz Castro, Juan Romero *}

${ }^{a}$ RNASA LAB research group,Faculty of Computer Science. University of A Coruña Spain. adriancarballal@gmail.com,nererofer@gmail.com,iry69.2@gmail.com,maria.luz.castro@udc.gal, jjrnasa@gmail.com

\begin{tabular}{|l|l|}
\hline Graphical Abstract & $\begin{array}{l}\text { Abstract. } \\
\text { One of the main challenges in Artificial Intelligence is to } \\
\text { do creative tasks such us creating music, art or novel } \\
\text { ideas. But, even more "simple" task such us have aesthetic } \\
\text { taste is very hard to a computer system. In this paper we } \\
\text { will present some information about how to building a } \\
\text { system that learn to predict the aesthetic and quality } \\
\text { value. We will also show preliminary results into a } \\
\text { experiment where human evaluate different images based } \\
\text { on its quality and aesthetic value. Finally we will show } \\
\text { some of the possibilities of this technology. }\end{array}$ \\
\hline
\end{tabular}

\section{Introduction (optional)}

Several research groups have tried to create computer systems capable of learning the way a group of human beings perceive aesthetics, to make a generative system or use for the selection or an automatic order of images. Some authors, such as Datta et al. [1], Wang et al. [2], Ke et al. [3] and Luo et al. [4] have conducted studies on the automation of aesthetic classification with technical characteristics such as light, saturation, Rule of the Thirds, etc. Another type of studies were those of Cela-Conde et al. [5], Forsythe et al. [6] and Nadal et al. [7] with a small set of images, but evaluated under control by a group of people with experimental conditions. Due to different problem in these datasets, they cannot be used to train and test automatic prediction and classification of images. To solve the problems it has created a new dataset with images of high statistical consistency through the DPChallenge.com Portal. This new dataset was evaluated by two different populations, thus creating several models in Machine Learning for the automated prediction of aesthetic and quality value, as well as that of DPChallenge.com.

\section{Materials and Methods (optional)}

The new dataset consists of 1000 images from the DPChallenge web portal. It has been evaluated in 3 different ways: evaluation from the DPChallenge portal with at least 100 scores per image; an aesthetic evaluation conducted under controlled experimental conditions and a minimum of 10 votes per images; and a quality evaluation conducted with similar conditions. To characterize the images, a multipurpose image classifier called WND-CHARM was used. This classifier extracts a large set of image features that include polynomial decompositions, high-contrast features, pixel statistics, textures, etc. The performance of the models was evaluated through the correlation of Spearman's Correlation Coefficient (rho) and Pearson's correlation coefficient (Pearson's r). 


\section{Results and Discussion (optional)}

Our results showed that the values for any of the 3 cases are below 0.6 on average; being 0.578 for DPChallenge, 0.456 for aesthetics and 0.539 for quality using Spearman's rho and $0.574,0.451$ and 0.562 respectively using Pearson's r.

\section{Conclusions (optional)}

The results show that in specific cases the images of DPChallenge are evaluated by different parameters: originality, quality, aesthetics, photo editing, etc. Regarding the use of complex systems to predict each of the three criteria, the highest one had a correlation of 0.578 using SVM. This value is similar to those obtained by Marin and Leder [8] using as criteria "arousal" (Spearman's rho $=0.44$ ) and "pleasantness" (Spearman's rho $=0.64$ ) or Nadal [7] with "beauty" (Spearman's rho $=0.648$ ) under experimental conditions with similar humans. Regarding the correlation between the SVM model with quality and individual aesthetics. It can be deduced that for the system it is simpler to learn quality values than aesthetic ones, since the second one is more subjective and less related to the characteristics of the image.

\section{References (mandatory)}

[1] R. Datta, D. Joshi, J. Li, J. Z. Wang, Studying aesthetics in photographic images using a computational approach, in: A. Leonardis, H. Bischof, A. Pinz (Eds.), Computer Vision - ECCV 2006, Springer Berlin Heidelberg, Berlin, Heidelberg, 2006, pp. 288-301.

[2] W. Wang, D. Cai, L. Wang, Q. Huang, X. Xu, X. Li, Synthesized computational aesthetic evaluation of photos, Neurocomput. 172 (C) (2016) 244-252.

[3] Y. Ke, X. Tang, F. Jing, The design of high-level features for photo quality assessment, in: 2006 IEEE Computer Society Conference on Computer Vision and Pattern Recognition (CVPR'06), Vol. 1, 2006, pp. 419-426.

[4] L.-K. Wong, K.-L. Low, Saliency-enhanced image aesthetics class prediction, in: 2009 16th IEEE International Conference on Image Processing (ICIP), 2009, pp. 997- 1000.

doi:10.1109/ICIP.2009.5413825.

[5] C.J. Cela-Conde, F.J. Ayala, E. Munar, F. Maestú, M. Nadal, \& M.A. Capó (2009). Sexrelated similarities and differences in the neural correlates of beauty. Proceedings of the National Academy of Sciences of the United States of America, 106, 3847-3852.

[6] A. Forsythe, M. Nadal, N. Sheehy, C.J. Cela-Conde, M. Sawey. (2011). Predicting beauty: Fractal dimension and visual complexity in art. British Journal of Psychology, 102, 49-70.

[7] M. Nadal, E. Munar, G. Marty, C.J. Cela-Conde (2010). Visual complexity and beauty appreciation: Explaining the divergence of results. Empirical Studies of the Arts, 28, 173-191.

[8] M. Marin, and H. Leder (2013). Examining complexity across domains: relating subjective and objective measures of affective environmental scenes, paintings and music. PLoS One, 8(8), e72412. 